\title{
3G Medicine - The Integration of Technologies
}

\author{
Andy Marsh \\ VMW Solutions \\ 9 Northlands Road, Whitenap, Romsey, Hampshire SO51 5RU, UK. \\ Tel : +44 7703235307 Fax : +44 1794522558 \\ e-mail andy.marsh@vmwsolutions.com
}

\begin{abstract}
Maturing telemedicine technologies, struggling mobile networking revenues and increased personal healthcare awareness have provided the foundations for a new market niche that of ' $3 \mathrm{G}$ Medicine'. During the last 5 years telemedicine (based on internet and web technologies) is becoming a reality both in terms of developing technologies and supportive legislation. Within Europe wireless infrastructures (3G Networking) has received a huge investment and although not well defined in how it will be achieved healthcare has been identified as a major stream of revenue with personal healthcare (e.g. EHCR on the handset) being a key issue especially for the handset manufactures. Combined with an increased awareness not only for outpatients but also for the "well-worried" (healthy and health conscious) 3G Medicine Services will play an important role in personal healthcare management. This paper presents a categorization of $3 \mathrm{G}$ Medicine services and the need to integrate technologies.
\end{abstract}

\section{Introduction}

During the last 5 years telemedicine has utilized developing technologies and matured into a now usable service acceptable both by patients and medical staff. In essence telemedicine supports the remote application of healthcare services. Isolated medical centers can be connected to hospitals, ambulances can transmit vital sign data to awaiting emergency units, General Practitioners can be keep informed of hospitalized patients and outpatients can be monitored whilst at home. By utilizing the latest mobile technologies (i.e. 3G networking) a new collection of "mobile telemedical" services can be developed referred to hereafter as "3G Medicine". These new services will support not only home care services but also mobile care services for example an outpatient may go about their daily business but still have the confidence they are being continuously monitored.

Traditionally, medical monitors were limited to data acquisition, typically implemented as Holters. Holters are used for 24-48 hour monitoring of ECG, EEG or polysomnography (EEG, EOG, EMG, EKG, heart rate, breathing, body position, snoring, etc.) and recording on cassette tape or flash memory. Recorded signals are then analyzed off line using dedicated diagnostic systems. Increased intelligence coupled with the low power consumption of the new generations of microcontrollers/DSPs combined with wireless technologies makes possible a whole 
range of intelligent monitor applications. Combined with an increased awareness not only for outpatients but also for the "well-worried" (healthy and health conscious) 3G Medicine Services will play an important role in personal healthcare management. Subsequently, the development of supportive 3G Medicine products and services will also create a new niche market economy for companies, especially SME's, to develop a range of collaborative technologies. The rationale of this paper is to identify a collaborative framework of integrated technologies and to highlight the requirement of a '3G Medicine Special Interest Group (SIG)' that will ensure conformity for the interoperability of $3 \mathrm{G}$ Medical products and services.

The major technologies that need to be integrated are presented in section 2 and a categorization of $3 \mathrm{G}$ Medical services is presented in section 3. However, for these new services to be generally accepted and used a certification and conformance body needs to be established as discussed in section 4. Conclusions are drawn in section 5 .

\section{The need to integrate technologies}

\subsection{Mobile Wireless Communications}

The choice of a wireless technology for a particular application depends on factors such as the range over which the device is expected to operate, the number of other wireless devices that are expected to operate within the same area, and the velocity of the device (that is, whether it is being used by someone sitting in a home or someone in a car traveling down a highway). Wireless technologies tend to be categorized based on the range in which they operate:

1. within a building such as a home or business (Pico cell);

2. within a neighbourhood (micro cell);

3. within a suburban area (macro cell);

4. across the globe.

Technologies And Services Existing Today. Many second-generation mobile technologies exist today each having influence in specific parts of the world. GSM, TDMA (IS 136), and CDMA (IS 95) are the main technologies in the secondgeneration mobile market. GSM by far has been the most successful standard in terms of it's coverage. All these systems have different features and capabilities. Although both GSM and TDMA based networks use time division multiplexing on the air interfaces, their channel sizes, structures and core networks are different. CDMA has an entirely different air interface.

Technologies And Services Existing Tomorrow. 3rd Generation Wireless, or 3G, is the generic term used for the next generation of mobile communications systems. $3 \mathrm{G}$ systems aim to provide enhanced voice, text and data services to user. The main benefit of the $3 \mathrm{G}$ technologies will be substantially enhanced capacity, quality and data rates than are currently available. This will enable the provision of advanced services transparently to the end user (irrespective of the underlying network and technology, by means of seamless roaming between different networks) and will bridge the gap between the wireless world and the computing/Internet world, making 
inter-operation apparently seamless. The third generation networks should be in a position to support real-time video, high-speed multimedia and mobile Internet access. All this should be possible by means of highly evolved air interfaces, packet core networks, and increased availability of spectrum. Although ability to provide high-speed data is one of the key features of third generation networks, the real strength of these networks will be providing enhanced capacity for high quality voice services. The need for landline quality voice capacity is increasing more rapidly than the current 2 nd generation networks will be able to support. High data capacities will open new revenue sources for the operators and bring the Internet more closer to the mobile customer. The use of all-ATM or all-IP based communications between the network elements will also bring down the operational costs of handling both voice and data, in addition to adding flexibility. The drive for $3 G$ is the need for higher capacities and higher data rate. Technologies like GPRS (General Packet Radio Service), High Speed Circuit Switched Data (HSCSD) and EDGE fulfill the requirements for packet data service and increased data rates in the existing GSM/TDMA networks. GPRS is actually an overlay over the existing GSM network, providing packet data services using the same air.

\subsection{Short-Range Wireless Connectivity}

Communication between various devices makes it possible to provide unique and innovative services. Although this inter-device communication is a very powerful mechanism, it is also a complex and clumsy mechanism, leading to a lot of complexity in the present day systems. This makes networking not only difficult but limits its flexibility as well. Many standards exist today for connecting various devices. At the same time, every device has to support more than one standard to make it inter-operable between different devices. Take the example of setting up a network in offices. Right now, entire office buildings have to make provisions for lengths of cable that stretch kilometers through conduits in the walls, floors and ceilings, to workers' desks. In the last few years, many wireless connectivity standards/technologies have emerged. These technologies enable users to connect a wide range of computing and telecommunications devices easily and simply, without the need to buy, carry, or connect cables. These technologies deliver opportunities for rapid ad hoc connections, and the possibility of automatic, unconscious, connections between devices. They will virtually eliminate the need to purchase additional or proprietary cabling to connect individual devices, thus creating the possibility of using mobile data in a variety of applications. Wired LANs have been very successful in the last few years and now with the help of these wireless connectivity technologies, wireless LANs (WLAN) have started emerging as a much more powerful and flexible alternatives to the wired LANs. Until a year ago, the speed of the WLAN was limited to $2 \mathrm{Mbps}$ but with the introduction of these new standards, we are seeing WLANs that can support upto $11 \mathrm{Mbps}$ in the ISM band. There are many such technologies/standards and notable among them are Bluetooth, IrDA, Home RF and IEEE 802.11. These technologies compete in certain fronts and are complementary in other areas. So, given the fact that so many technologies exist, which technology is the best and which solution should one select for a specific application? The premise behind all these standards is to use some kind of underlying 
radio technology to enable wireless transmission of data, and to provide support for formation of networks and managing various devices by means of high level software.

\subsection{Wireless terminals}

There is going to be an estimated 60 Million Mobile Internet users in 2004, according to estimates, and this emerging market is now starting to take off. All different parts of the software and Internet industry is moving in to this space with a huge increase in interest over the last 12 months. The recent JavaOne conference in San Francisco also showed this trend, wireless is a major opportunity for Java to make it big. There are lots of debates about Java or NoJava, EPOC vs PalmOS vs PocketPC etc. and maybe these debates are made obsolete by Java? No matter what, there is still one thing that remains to be determined: What are the devices that we all will use?

The Wireless Application Protocol. The mass market device for the Mobile Internet applications is likely to be the phone with a WAP browser. Most vendors will include WAP in all their phones. This means that anyone that buys a phone to use for talking is getting the Mobile Internet features built in. WAP might not have the most advanced applications at this point, but it is very easy to use and get into (especially when GPRS takes away the connection setup-time). Even with the advent of more advanced applications, WAP will still be used to access information, much like the web browser on the PC. The main difference is that phones are not going to be used that much for random browsing, but rather information access, straight to the point. With the advent of WAP 2.0, where multimedia and streaming is foreseen, the width of applications increases drastically. The Wireless Application Protocol is a standard developed by the WAP Forum, a group founded by Nokia, Ericsson, Phone.com (formerly Unwired Planet), and Motorola. WAP defines a communications protocol as well as an application environment. In essence, it is a standardized technology for cross-platform, distributed computing. WAP is very similar to the combination of HTML and HTTP except that it adds in one very important feature: optimization for low-bandwidth, low-memory, and low-display capability environments. These types of environments include PDAs, wireless phones, pagers, and virtually any other communications device.

JAVA(TM) wireless toolkit. Sun Microsystems on Nov. 27, 2001 announced that it is offering an enhanced version of the Java (TM) 2 Platform, Micro Edition (J2ME(TM)) Wireless Toolkit(). Live demonstrations of the toolkit, including the early access Japanese version, was presented at the JavaOne(SM) Conference Yokohama, November 28-30, 2001. The updated toolkit securely integrates with major Java technology Integrated Development Environments (IDEs) and is the first toolkit to deliver a set of enhanced new features that enable the design and implementation of Over-The-Air (OTA) provisioning for mobile devices. OTA provisioning is the wireless delivery of applications and services over a secure network to mobile devices, including mobile handsets and PDAs. Named this June by JavaWorld.com as the "Best Java Device Application" by JavaWorld.com, the J2ME Wireless Toolkit will help generate more rich and innovative applications and services for Java technology-enabled devices. 


\subsection{Information-Standards Organisations in Healthcare Informatics}

The necessity of having standards is well understood and highly appreciated. A number of standardisation organisations and committees have been quite active in the development of standards that relate to healthcare informatics:

- American National Standards Institute (ANSI)

- CEN (Comité Europeén de Normalisation) Technical Committee 251

- ISO Technical Committee 215

- American Society for Testing and Materials Committee E31 (ASTM E31)

- Healthcare Informatics Standards Board (HISB)

- Computer-Based Patient Record Institute (CPRI.

\subsection{Security}

The explosive growth in computer systems and their interconnections via networks, mainly the Internet, has increased the dependence of both organizations and individuals on the information stored and communicated using these systems. This, in turn, has led to heightened awareness of the need to protect data and resources from disclosure, to guarantee the authenticity of data and messages and to protect systems from network-based attacks.

\subsection{Wireless medical devices}

For millions of Europeans with chronic medical conditions, careful day-to-day health monitoring can help avert catastrophe. Home health gauges abound, but they take readings only at discrete points in time and require a patient's active participationanswering a computer questionnaire, for example. The ideal monitor, however, would record data constantly, and patients wouldn't even notice it's there namely wearable health sensors. "There's no question we're going remote, and we're going wireless," says Credit Suisse First Boston's Robert Hopkins. A non-invasive ambulatory monitoring system can provide clinically relevant parameters with the capability to perform continuous monitoring over hours, days and weeks thus providing a "movie" of health and/or disease versus the "snapshot" resulting from a standard history and physical examination conducted in a physician's office. An ambulatory system could be used to collect and store cardiac, respiratory, blood pressure, posture, activity and emotional measures.

\subsection{Wireless healthcare software services}

The technology sector of healthcare is entering into a new evolutionary phase. The medical community has an obligation to the public to provide the safest, most effective healthcare delivery system possible. Optimal medical practice will depend on computers. Outcomes analysis that will shape medical treatments in the future will be derived directly from the data entered into Windows Powered mobile devices. If a global database could be obtained using point-of-care devices, existing methods for tracking clinical outcomes would become obsolete. Optimal management of disease 
prevention and management could be achieved! In addition, these devices, with appropriate software, will allow for foolproof coding and billing. Using Windows Powered mobile solutions, physicians and hospitals can capture additional earned revenue that can amount to millions of lost healthcare dollars per year.

\section{A framework for mobile medical services}

Based upon the authors experience in identifying a framework for internet/web-based telemedicine [1], a framework for Mobile (M-) medical services has been defined [2] that contains three categories of services namely: M-Safety, M-Healthcare and MMedicine. (To support these services there needs to be an investment in hardware R\&D to develop the supportive M-Platforms and M-Sensors and also software R\&D to incorporate M-Security and Compunetics)

\subsection{M-Safety services}

The M-Safety (Mobile-Safety) services are a form of the newly developing Mobile Location Services (MLS) but focus on the potential safety implications of location awareness and include child monitoring, location advisory and third party location monitoring services. Three sub-categories of M-Safety services have been identified, namely:

- where the user of the service is informed where they are.

- where another person is informed where the user of the service is.

- where a 3rd party monitors the location of the user of the service.

M-Safety service Category 1:(Pharos). By utilizing the latest mobile phone handsets, with locatable functionalities, a range of services can be developed that verbally informs the user where they are in terms of street names and nearest points of interest. Defined originally for the visually impaired to act as a guidance tool the "Pharos" service is equally applicable in any outdoor Location Based Navigation \& Direction environment.

M-Safety service Category 2:(MINDER). By utilizing the latest locatable technologies a range of services can be developed whereby the location of the user is illustrated on a digital map that can be viewed by a web browser. Defined originally for monitoring the movements and whereabouts of children, but equally applicable to any outdoor tracking environment, the " MINDER - Movement INformation DElivered Regularly" service provides regular updates of the outdoor position, displayed on a digital street map, of a personal locatable tag.

M-Safety service Category 3:(Guardian). By utilizing the latest developments in GPS and digital compass technologies a range of services can be developed whereby the indoor location of a user can also be monitored by a 3rd party service provider. Defined originally for the monitoring of the elderly, but equally applicable in any indoor tracking environment, the "Guardian" service [2] provides a reassurance 
mechanism for family members that for example an elderly people has not fallen over or has become trapped etc.

\subsection{M-Healthcare services}

The M-Healthcare (Mobile-Healthcare) services are a subset of the newly developing 3G Medical services and focus on the collection and interpretation of personal medical sensor data. a range of M-Healthcare services can be developed which include the recording of personal sensor data (for example ECG) for future comparison, a mechanism to check the data for warning signs (for example high blood pressure) and an automated analysis of the sensor data. Three sub-categories of M-healthcare services have been identified, namely:

- where the results of personal sensors are recorded for later use.

- where an early warning system checks the personal sensor results.

- where the results of personal sensors are automatically diagnosed.

M-Healthcare service Category 1:(MEMOIR). By using the memory capabilities of mobile phone handsets a range of services can be developed that record the personal sensor data that can then be used for later comparisons. Defined originally for monitoring ECG over 24 hours, the "MEMOIR services [2] are equally applicable to monitoring any sensor data over long time periods.

M-Healthcare service Category 2:(LOCUM). By using the latest developments in mobile handset technologies that support computational facilities (e.g. Java on the handset) a range of customizable personal early warning services can be developed whereby the data of the users sensors are analyzed against personalized predefined values. If a discrepancy occurs the user is subsequently warned. Defined originally for monitoring the blood pressure of the elderly, but equally applicable in any high risk category, the "LOCUM - LOcally Controlled Ubiquitous Monitoring" service [2] provides a personal healthcare reassurance mechanism.

M-Healthcare service Category 3:(NOMADS). By using the latest developments in wireless communication technologies (such as GPRS) a range of NOn intrusive Monitoring and Automated Diagnosis Services (NOMADS) can be developed whereby the data of the users sensors are remotely analyzed. If a problem is diagnosed then a member of the medical community is alerted and the appropriate action initiated. Defined originally for the monitoring of oncology patients the "NOMADS" service [2] is equally applicable for any outpatient analysis.

\subsection{M-Medicine services}

The M-Medicine (Mobile-Medicine) services are a subset of the newly developing 3G Medical services and focus on 2-way communication between diagnostic medical servers (supported by medical staff) and the users medical sensors. A range of MMedicine services can be developed which support personalized care (for example informing the user then checking that drugs have been taken with the correct dosage 
and at the correct time), personalized nursing (for example altering a drug prescription due to updated sensor data) and personalized doctoring (for example modifying a treatment plan). Three sub-categories of M-Medicine services have been identified, namely:

- where the user is informed to do an action that is monitored.

- where the action to be undertaken by the user is modified.

- where the actions to be undertaken by the user are defined.

M-Medicine service Category 1:(3G-Care). By using the latest developments in 3G technologies a range of 3G-Care services can be developed whereby medical staff can remotely instruct and monitor users medical sensors and related supported devices. Defined originally for monitoring drug usage, the "3G-Care " services [2] are equally applicable for instructing the user and monitoring any sensor and/or related supported device usage.

M-Medicine service Category 2:(3G-Nurse). By using the latest developments in $3 \mathrm{G}$ technologies a range of $3 \mathrm{G}-$ Nurse services [2] can be developed whereby medical staff can remotely instruct, monitor and control users medical sensors and related supported devices enabling modifications of personalized treatment plans and early warning symptoms.

M-Medicine service category 3:(3G-MD). By using the latest developments in $3 \mathrm{G}$ technologies a range of 3G-MD services [2] can be developed whereby the Medical Doctor can define a personalized treatment plan and early warning symptoms that can then be downloaded to the users medical sensors and related supported devices.

\section{The need for conformance}

\subsection{A US perspective}

The HMO's, that have supporting legislation, have driven the adoption of telemedicine within the US. Internet based applications are being used to improve access to care and the quality of care, reducing the costs of care and the sense of professional isolation for some healthcare practitioners. In this environment the introduction of wireless telemedicine should be introduced via the HMO's expanding their range of services and therefore compatible with the presently installed systems and envisaged wireless LAN systems. In summary, it is envisaged that the introduction of 3G Medical services in the US will again be driven from the HMO's therefore it is essential that some form of standardization and conformance be undertaken in conjunction with existing telemedical services.

\subsection{A European perspective}

Within Europe telemedicine has not been driven so much by HMO's but more by isolated medical institutions and regional trails. The legislation aspect of telemedicine also within Europe is more complex than in the US especially when National 
boundaries have to be crossed. However, the telecommunications markets in Europe are more focused and standardized than their US counterparts and it is this that is envisaged to be the driving force behind the introduction of $3 \mathrm{G}$ medical services in Europe. In summary, it is essential that some form of standardization and conformance be undertaken in conjunction with developing telecommunication infrastructures and services.

\subsection{The 3G Medicine Special Interest Group}

By combining the two perspectives above it is clear that for $3 \mathrm{G}$ Medicine services to be generally available and accepted worldwide then there needs to a standardization and/or conformance certification group. Similarly to the bluetooth Special Interest Group it is therefore proposed that a 3G Medicine Special Interest Group (SIG) be established with representatives from both HMO's and telecommunications domains both in Europe and the US. Additionally there also needs to be representatives from a number of supporting industrials including platform developers, compunetics (computing and networking) suppliers, security advisors, medical data sensor developers and service developers.

The objective of the 3G Medicine SIG is to combine 20 areas of expertise:

1. Wireless Medical devices (ERM 11. Compression (JPEG 2000, Wavelet) TG30)

12. Archival (HIS, Data warehousing)

2. Mobile Terminals (PDA, Smart phone)

13. Knowledge discovery (personalized alarms)

3. Operating Systems (Linux, Palm OS)

4. Data Storage (M-EHCR)

14. Healthcare providers (Doctors, Nurses)

5. Data Encoding (XML, WML)

6. Programming environments (JAVA)

15. Personal healthcare management providers

7. Visualization (MPEG, VRML)

16. Standardization (R\&TTE)

8. Transmission (GPRS, EDGE, UMTS)

17. Conformance (FDA, EU CE Marking)

9. Collaboration (SMS, WAP, HTTP)

10. Privacy \& security (TLS, SSL, PKCS)

18. Legislation (National, EU polices)

19. Service providers (HMO's)

20. User groups (Elderly, Outpatients)

The 3G Medicine SIG will therefore tackle such issues as device availability, possibilities for health with $3 \mathrm{G}$ networking, the services that will be required (by health professionals, ambulatory, patients and citizens), the applications that will be developed, the costs (private and public) and compliance with technical issues, legislation and regulatory frameworks. 


\section{Conclusions}

For the successful development of $3 \mathrm{G}$ Medical services there needs to be an agreement on what types of categories of services will become available, how different complementary industries can work together to develop these services and how the developed services are certified. The rationale of this paper was to suggest such a framework. In the first instance the author has categorized potential 3G medical services into 3 groups namely M-Safety, M-Healthcare and M-Medicine. Each group is subdivided further relating to how the medical data is utilized. To support the development of these services the author has also identified the necessity of M-Sensors, M-Platforms, M-Compunetics and M-Security. To ensure conformance the author has also suggested that in the first instance a Special Interest Group be established to co-ordinate the development of 3G Medical services and provide certification of developed services.

\section{References}

1. Marsh,A, Grandinetti,L, Kauranne, T, "Advanced Infrastructures for Future Healthcare", IOS press, 2000.

2. www.vmwsolutions.com

3. www.benefon.com

4. Marsh, A, May, M., Saarelainen,M., "Pharos: Coupling GSM \& GPS-TALK technologies to provide orientation, navigation and location-based services for the blind", IEEE ITAB-ITIS 2000, Washington Nov, 2000.

5. Strouse, K, "Strategies for success in the new telecommunications marketplace", Artech House, USA, 2001.

6. PriceWaterhouseCoopers, "Technology Forecast 2001-2003, 2001

7. Dornan,A, "The essential guide to wireless communications applications", Prentice Hall, USA, 2001.

8. Jupiter Research, "Mobile Commerce Report", 2001.

9. Scheinderman, R, "It's time to get smart", Portable design, March 2001, pp22-24.

10. ARC Group, “3G Industry Survey”, 2001 